\title{
THE NEUTRAL ENVELOPES OF PLANETARY NEBULAE: MOLECULES AND H I
}

\author{
P.J. HUGGINS \\ Physics Department, New York University, 4 Washington Place, New York, NY 10003, U.S.A.
}

\begin{abstract}
This paper summarizes recent developments in the study of planetary nebulae using observations of molecular lines and the $21 \mathrm{~cm}$ line of HI. The observations reveal that many planetary nebulae are surrounded by envelopes of neutral gas, whose mass often exceeds that of the ionized nebulae. They also provide valuable information on the physical and chemical properties of the envelopes, their structure, and kinematics. The neutral envelopes firmly link the formation of planetary nebulae with the mass loss by AGB stars, and can play an important role in the subsequent evolution of the nebulae.
\end{abstract}

\section{Introduction}

Rapid progress has been made during the last few years in the study of the neutral envelopes of planetary nebulae $(\mathrm{PNe})$. The envelopes of $\approx 70 \mathrm{PNe}$ have now been detected in the lines of one or more molecular species or $\mathrm{HI}$. They are found in compact $\mathrm{PNe}$ (e.g., $\mathrm{BD}+30^{\circ} 3639$ and IC 4997) as well as much more evolved PNe (e.g., the Dumbbell and Helix nebulae), and sufficient data have now been obtained that some general conclusions can be made about the properties of the envelopes and their role in the formation and evolution of the ionized nebulae.

The development of this field confirms earlier ideas, based on indirect evidence, that many, if not most PNe must be surrounded by envelopes of neutral gas. The concept of ionization bounded nebulae has a long history in the study of $\mathrm{PNe}$, and the mass-radius relation, first discussed by Pottasch (1980), strongly suggested the presence of envelopes of neutral gas which become ionized as the nebulae expand. The typical ionized mass of an evolved PN is about $0.3 M_{\odot}$; on the basis of the mass-radius relation, one might expect roughly this amount of neutral matter around a compact $\mathrm{PN}$, and in some objects this turns out to be the case. This amount of cool, neutral matter at representative distances of $0.5-10 \mathrm{kpc}$, is, in fact, quite difficult to detect in $\mathrm{H} \mathrm{I}, \mathrm{H}_{2}$, or in a trace species, except under special circumstances. Accordingly, the development of this field has, to a large extent, paralleled the capabilities of the available instrumentation.

The data on the neutral envelopes of PNe have grown dramatically since the earlier reviews of this field by Black (1983) and Rodriguez (1989). The volume of recent data means that the present paper is, necessarily, highly condensed, and a more extended discussion of some of the issues raised here is given by Huggins (1992). 
Table 1. Main Probes of the Neutral Envelopes of PNe

\begin{tabular}{lcccc}
\hline Species & Wavelength & Detections & Comments & References \\
\hline $\mathrm{H} \mathrm{I}$ & $21 \mathrm{~cm}$ & 10 & mainly in absorption & 1 \\
$\mathrm{H}_{2}$ & $2.1 \mu \mathrm{m}$ & 33 & thermal? E/k $\approx 6700 \mathrm{~K}$ & 2 \\
$\mathrm{CO}$ & $1.3,2.6 \mathrm{~mm}$ & 45 & thermal E/k=6,16 K & 3 \\
$\mathrm{OH}$ & $18 \mathrm{~cm}$ & 13 & maser & 4 \\
$\mathrm{HCN}$, etc. & $\mathrm{mm}$ & 5 & thermal & 5 \\
OI etc. & opt./ir & 5 & NaI/OI,CII (ir PDR lines) & 6
\end{tabular}

1. Taylor et al. (1990) Gussie (1992) 2. Webster et al. (1988) Zuckerman \& Gatley (1988) 3. Huggins \& Healy (1989) Huggins et al. (1992a) 4. Zijlstra et al. (1989) 5. Cox et al. (1992) 6. Dinerstein (1991)

\section{Observations}

The principal atomic and molecular species which have been used to study the neutral envelopes of $\mathrm{PNe}$ are listed in Table 1, together with the approximate number of $\mathrm{PNe}$ detected in each. The envelopes which have been mapped in one or more lines are listed in Table 2. The references in the tables are to recent or summary papers from which other contributions can be found, and are the main references for the discussion which follows. The typical angular resolution of the maps is $3^{\prime \prime}-30^{\prime \prime}$, and the observations of the millimeter and radio lines (but not the infrared lines) usually provide high velocity resolution $(\approx 1 \mathrm{~km}$ $\mathrm{s}^{-1}$, or better) with which the kinematics can be studied.

The information obtained from the various lines of the neutral species is, to a large extent, complementary. The millimeter lines of $\mathrm{CO}$ are the most useful general probes of the molecular gas, and have been the most widely observed; the IRAM 30-m telescope has proved to be particularly effective for these studies. The infrared $\mathrm{H}_{2}$ lines have been detected in nearly as many $\mathrm{PNe}$ as $\mathrm{CO}$, but their interpretation is still not entirely clear; the emission arises from high lying energy levels which may be excited in shocks, or by ultraviolet radiation or collisions at high temperatures in the photodissociation regions (PDRs) formed by the central star. The relative importance of these processes in most PNe has not yet been sorted out (e.g., Dinerstein 1991, Huggins 1992, Tielens 1992). There is, however, a good correlation between the PNe which have been detected in $\mathrm{CO}$ and $\mathrm{H}_{2}$. The data on other molecules and atoms is less extensive, but is nevertheless important for outlining a more complete picture.

Certain general features of the neutral envelopes are readily apparent from the observations. Among the most important is the continuity between AGB envelopes and PNe implied by the $\mathrm{CO}$ and $\mathrm{OH}$ observations. For example, $\mathrm{OH}$ maser emission can be seen in $\mathrm{O}$-rich AGB envelopes, proto- $\mathrm{PNe}$, and young $\mathrm{PNe}$; and in $\mathrm{Vy} 2-2$ and $\mathrm{OH} 0.9+1.3$, which have been mapped at high resolution (Table 2), the $\mathrm{OH}$ surface brightness and the shell sizes $\left(0.6-3 \times 10^{16} \mathrm{~cm}\right)$ are characteristic of AGB stars. The relatively small dimensions of the maser shells means that they will, of course, be rapidly destroyed by the developing nebulae. $\mathrm{HI}$ is seen in young PNe, but rarely in AGB envelopes, so is likely to be produced as a result of the evolution of the central star. Perhaps the most surprising feature is the survival of substantial amounts of molecular gas in the envelopes of some very evolved $\mathrm{PNe}$ (e.g. the Dumbbell, and the Helix). Specific aspects of the envelopes are discussed in the sections that follow. 
Table 2. Maps of the Neutral Envelopes of PNe

\begin{tabular}{lll}
\hline Species & \multicolumn{1}{c}{ PNe } & Refs \\
\hline H I & BD+30³639, IC 418, NGC 6302 & $2,2,1$ \\
$\mathrm{H}_{2}$ & CRL618, He2-111, He2-114, Hf48, IC 4406, Mz1 & $1,3,3,3,1,3$ \\
& NGC 2346, NGC 2818, NGC 2899, NGC 3132 & $4,3,3,1$ \\
& NGC 4071, NGC 5189, NGC 6445, NGC 6720 & $3,3,3,4 \& 5$ \\
& NGC 6772, NGC 6781, NGC 6853, NGC 7027 & $3,4,4,1$ \\
CO & CRL618, IC 4406, IRAS 21282+5050, M1-7, M2-51 & $6,7 \& 9,6,6 \& 8,8$ \\
& M4-9, NGC 2346, NGC 3132, NGC 6072, NGC 6563 & $8,10,7,9,9$ \\
& NGC 6720, NGC 6772, NGC 6781, NGC 7027 & $10,10,10,12$ \\
OH & NGC 7293, VV47 & $11 \& 8,10$ \\
HCN etc. & CRL618 (HCN), NGC 7027 (HCO+) & $15,14,13,13$ \\
\hline
\end{tabular}

1. References prior to 1988 can be traced through the review by Rodriguez (1989) 2. Taylor et al. (1989, 1990) 3. Webster et al. (1988) 4. Zuckerman \& Gatley (1988) Zuckerman et al. (1990) 5. Greenhouse et al. (1988) 6. Shibata et al. (1992) 7. Sahai et al. (1990, 1991) 8. Forveille \& Huggins (1991) 9. Cox et al. (1991) 10. Bachiller et al. (1989a, 1989b, 1992) 11. Healy \& Huggins (1990) 12. Bieging et al. (1991) 13. Shepherd et al. (1990) 14. Payne et al. (1988) 15. Seaquist et al. (1991) 16. Neri et al. (1992) 17. Likkel (1992) See also Clegg et al. and Sahai et al. in these proceedings

\section{The Physical and Chemical Properties of the Envelopes}

\subsection{The Molecular Gas}

The structure of the molecular component of the neutral envelopes is well documented in the $\mathrm{CO}$ and $\mathrm{H}_{2}$ maps referenced in Table 2. In general, the large scale structure of the envelopes shows a good deal of symmetry, and is dominated to a greater or lesser extent by the degree to which the matter is concentrated in a preferred plane, or equator. In some compact PNe (e.g., NGC 7027, or CRL 618), the CO envelope is fairly complete, and is considerably more extended than the ionized nebula, consistent with the idea that the central star has very rapidly evolved from the mass losing AGB phase, and that the young nebula has formed with the outer envelope still intact. In more evolved PNe, the gas is primarily found in toroidal structures around the waist of the nebula, in thin shells, or in isolated condensations. Among the best examples are: NGC 6720 and NGC 6781, where the molecular gas is in thin, ellipsoidal shells; the bow-tie PN NGC 2346, where the gas lies around the waist and to some extent along the walls of the bow tie; and VV 47, where the gas is found within the bright, symmetric, optical lobes. The optical morphology of the $P N e$ with neutral envelopes clearly reflects the underlying structure of the envelopes.

The available maps also show that the molecular gas in the envelopes is not homogenous, but is clumped on the smallest size scales observed. This is the case in essentially all of the PNe which have been well resolved in $\mathrm{CO}$, ranging from the extended envelope of the compact PN NGC 7027 to the thin shells of the much more evolved Helix nebula; in these two cases the clump scale size is $\lesssim 0.02-0.01$ pc. An extreme form of clumping is seen in the numerous cometary globules inside the ionized cavity of the Helix, where the optical condensations are $\approx 1$ " in size; recent observations show that these, too, have cores of molecular gas (Huggins et al. 1992b). 
The physical conditions in the molecular gas have been studied in several PNe using the ratios of the rotational lines of $\mathrm{CO}$ (e.g. Bachiller et al. 1992). The excitation temperatures are typically $\approx 20 \mathrm{~K}$, and the densities are rather high $\left(\gtrsim 3 \times 10^{3} \mathrm{~cm}^{-3}\right.$, and possibly much higher). The infrared $\mathrm{H}_{2}$ lines are not excited under these conditions, but require high temperature regions due to shocks or PDRs. In line with this, the mass of excited $\mathrm{H}_{2}$ is invariably found to be small $\left(\lesssim 1 \times 10^{-3} M_{\odot}\right)$ and is much less than the total mass of molecular gas in the envelopes. Under the conditions found in the bulk of the molecular gas, the $\mathrm{CO}(2-1)$ line provides a useful estimate of the total mass, whose main uncertainty (apart from the distance) is the $\mathrm{CO} / \mathrm{H}$ ratio (Huggins \& Healy 1989); using the $\mathrm{C}$ or $\mathrm{O}$ abundance from the ionized gas then gives a good lower limit to the total mass. With this approach, the molecular envelopes detected in $\mathrm{CO}$ have masses in the range $1-1 \times 10^{-3} M_{\odot}$; more detailed estimates are available in a few cases, e.g., Jaminet et al. (1991) find the mass of molecular gas is $1.4 M_{\odot}$ in NGC 7027. Upper limits on nearby PNe without CO emission are typically $\lesssim 1 \times 10^{-2} M_{\odot}$. There is, therefore, a very large range in the mass of molecular gas in PNe; even in some old PNe (e.g. NGC 6781 and the Helix) the mass of molecular gas is roughly comparable to the mass of ionized gas. The large variation found is attributable to a combination of population and evolutionary effects $(\S 4)$.

\subsection{The Atomic Gas}

There is considerably less data on the properties of the atomic component of the neutral envelopes. For nebulae which are optically thick, it is expected that the radiation field of the central star longward of the Lyman limit will establish a warm, mainly atomic PDR at the periphery of the ionized gas. Evidence for this comes from the study of the C II and O I infrared fine structure lines in NGC 7027 by Ellis and Werner (1985; see also Tielens 1992). Although the lines are not spatially resolved, the line ratios indicate a gas temperature of $500 \mathrm{~K}$, and a density of $7 \times 10^{4} \mathrm{~cm}^{-3}$, which are consistent with the idea that the lines originate in the PDR. The mass of this region (inferred from the $\mathrm{C} / \mathrm{H}$ ratio) is $0.3 M_{\odot}$, which is roughly that expected if the $\mathrm{PDR}$ extends for $\approx 1$ visual magnitude of extinction into the neutral shell. Further work has been reported by Dinerstein et al. (1991), who find roughly similar physical conditions in 4 additional PNe.

From direct observations of the $\mathrm{HI}$ gas using the $21 \mathrm{~cm}$ line, the location of the $\mathrm{HI}$ is known in only three cases, all young PNe (Table 2). In NGC 6302 the HI is found in the dark lane around the waist of the nebula, and in $\mathrm{BD}+30^{\circ} 3639$ the $\mathrm{H} \mathrm{I}$ is concentrated near the H II region, and may lie within the molecular shell (Bachiller et al. 1991). In these two cases the masses of $\mathrm{HI}$ and $\mathrm{H}_{2}$ are very roughly comparable, and it is likely that the $\mathrm{HI}$ is photo-produced from $\mathrm{H}_{2}$ in PDRs between the molecular and ionized gas. In IC 418 the situation is rather different. The $\mathrm{HI}$ emission is very extended $(0.4 \mathrm{pc})$ compared to the ionized nebula and the mass of the extended H I envelope $\left(0.4 M_{\odot}\right)$ is very much larger than the mass of the molecular component (based on $\mathrm{CO}$ ); thus most of the extended remnant wind is atomic. $\mathrm{HI}$ is also seen in several additional PNe in absorption against the continuum $\left(\mathrm{N}(\mathrm{HI})\right.$ is typically a few $\left.\times 10^{20} \mathrm{~cm}^{-2}\right)$, but others like NGC 7027 are not detected. Most of those detected have little or no molecular emission, so they, like IC 418, may belong to a population of $\mathrm{PNe}$ with relatively thin envelopes. For the PNe detected in H I, lower column densities are found in the larger PNe (Taylor et al. 1990), as might be expected from the expansion of the envelopes. Further work, however, is needed before a coherent picture of the atomic gas is developed. 


\subsection{Kinematics}

The kinematics of the neutral envelopes are important clues to the dynamical evolution of PNe. Although the typical expansion velocity of the ionized gas in a $P N$ is $\approx 20-25 \mathrm{~km}$ $\mathrm{s}^{-1}$, and that of an AGB envelope is $\approx 14 \mathrm{~km} \mathrm{~s}^{-1}$, much higher velocity molecular winds appear to be a fairly common feature of the intervening proto-PN phase. The highest velocity molecular wind is found in CRL 618, which has, in fact, already developed into a very young $P N$. It shows a normal, molecular, AGB envelope expanding at $19 \mathrm{~km} \mathrm{~s}^{-1}$, and a high velocity, bipolar, molecular wind expanding at $\approx 200 \mathrm{~km} \mathrm{~s}^{-1}$ (e.g., Neri et al. 1992). A number of other young $\mathrm{PNe}$ also show evidence for a high velocity molecular component, although are less spectacular than CRL 618. Some other young PNe with low velocity molecular envelopes show high velocity atomic jets (e.g., M1-16, Schwarz 1992). The origin of these high velocity phenomena are not well understood. They are clearly able to modify the structure of the envelopes, but their effects are not yet well documented.

In all the more evolved PNe with molecular emission or $\mathrm{HI}$ absorption, the typical expansion velocities of the bulk of the neutral gas are moderate, $\approx 15-30 \mathrm{~km} \mathrm{~s}^{-1}$, similar to typical PN expansion velocities. In a few cases where higher velocity wings are seen, mapping in $\mathrm{CO}$ shows that this can be attributed to wind velocities which increase towards the poles of a mainly equatorial distribution of matter. The best example of this is the Ring type PN, NGC 6781, where high quality CO mapping is well accounted for by a thin ellipsoidal shell with an expansion velocity which increases with latitude (Bachiller et al. 1992). In the most extended $\mathrm{PNe}$ where the individual condensations can be observed (e.g., the Helix and VV47), the CO linewidths are very small $\left(\approx 1 \mathrm{~km} \mathrm{~s}^{-1}\right.$; Forveille \& Huggins 1991).

The kinematic relation between the neutral and ionized gas is an important diagnostic of the shell development. In several PNe which have been well observed in $\mathrm{CO}$, the expansion velocity of the neutral envelope appears to be larger than that of the ionized gas, an extreme example being $\mathrm{BD}+30^{\circ} 3639$, where the $\mathrm{CO}$ expansion velocity is $52 \mathrm{~km} \mathrm{~s}^{-1}$ (Bachiller et al. 1991), and those of [O III], $\mathrm{H} \alpha$, and [N II] are 23-27 $\mathrm{km} \mathrm{s}^{-1}$. Thus the general trend for lines of lower ionization species to have larger expansion velocities, which is seen in the ionized nebulae, extends to some of the neutral shells as well. Unfortunately, few of the PNe which have been mapped in detail in $\mathrm{CO}$ have also been mapped in one or more of the ionic lines so that they can be compared in detail. One interesting exception is the Helix, where the CO around the ring structure observed by Healy \& Huggins (1990) appears to be kinematically part of a more complete shell of atomic gas seen in [N II] (Walsh \& Meaburn 1987). Further optical studies of the PNe whose neutral envelopes have been mapped in detail (Table 2) would be important for developing a clearer understanding of their dynamics.

\subsection{Chemistry}

An additional aspect of the envelopes in which recent progress has been made is the chemistry of the neutral gas. A rich chemistry takes place in the thick envelopes of AGB stars, and an increasing number of molecular species are also found in the much harsher environments of PNe. 14 species are detected in the very young PN CRL 618 (e.g., Bujarrabal et al. 1988) where the envelope still retains some of the AGB characteristics; fewer species are seen in more evolved objects, but five (CO, $\mathrm{HCN}, \mathrm{HNC}, \mathrm{CN}$, and $\mathrm{HCO}+)$ have now been detected in NGC 6072, and IC 4406 (Cox et al. 1992), where the central 
stars are on cooling tracks $\left(T \approx 100,000 \mathrm{~K}, L \approx 100-400 L_{\odot}\right)$ and the envelopes have developed well beyond the AGB phase.

Certain systematic trends in the abundances in the envelopes are already apparent from the data in hand, the most striking being a dramatic increase in the relative abundances of $\mathrm{HCO}+$ and $\mathrm{CN}$ as the PNe form (Cox et al. 1992). Interferometer maps also show that the $\mathrm{HCO}+$ in NGC 7027 is found in a thick torus around the periphery of the ionized gas (Likkel 1992, Deguchi et al. 1992). A range of different processes need to be considered in understanding the unusual abundances found in the envelopes. The shielding of dissociating UV radiation is an overriding consideration, and the strong clumping of the gas mentioned in $\S 3.1$ clearly plays a role, although the effects of UV radiation (and possibly x-rays) from the central star can also initiate the synthesis of some species under appropriate conditions. High temperature processing through shocks is also likely to be important, especially in the proto-PN and early PN phases. A clear picture has not yet emerged (Cox et al. 1992, Howe et al. 1992), in part because we do not understand in detail the physical conditions in the gas. As in AGB envelopes and the ISM, there is a strong probability that the chemistry in $\mathrm{PNe}$ will eventually prove to be invaluable in understanding other aspects of the envelopes.

\section{Evolution}

On the basis of the observational results described above it is possible to outline some aspects of the evolution of the envelopes of PNe. This is more easily done for the molecular gas component, since the data are more extensive. From the molecular line surveys it is clear that the possession of a substantial molecular envelope is related to the population type of the PN. The $\mathrm{H}_{2}$ emission (Webster et al. 1988, Zuckerman \& Gatley 1988) and CO emission (Huggins \& Healy 1989) is correlated with galactic latitude, bipolarity, and PN type, with the implication that higher mass AGB stars are those that form PNe with substantial molecular envelopes. Several factors probably contribute to this. The higher mass cores move more rapidly across the H-R diagram and form compact PNe while the envelope is still optically thick to dissociating radiation; their mass loss rates on the AGB are also probably larger, so the envelopes are thicker; and their tendency to bipolarity and filamentary structure provide additional shielding of the molecular gas.

It is also possible to study quantitatively the evolution of the molecular envelopes once the PNe have formed. Huggins \& Healy (1989) have shown that for PNe detected in CO, the ratio $M_{m} / M_{i}$ (where $M_{m}$ is the mass of the molecular gas, and $M_{i}$ is the mass of ionized gas) decreases systematically with increasing nebular size. This relation is shown in Fig. 1, and has recently been confirmed and extended (Huggins et al. 1992). The interpretation of this diagram is as follows. When the central stars first cross the H-R diagram and form compact $\mathrm{PNe}$, the system is still largely molecular $\left(M_{m} / M_{i} \gg 1\right)$. As the gas expands, the mass of ionized gas increases at the expense of the neutral envelope. The masses of ionized and molecular gas are roughly comparable by the time the $\mathrm{PN}$ has a radius of $\approx 0.1 \mathrm{pc}$, and only a residual amount of molecular gas remains when the $P N$ is several tenths of a parsec in size. The observed structure of the molecular envelopes at different stages of evolution (§3.1) strongly supports this evolutionary scenario. 


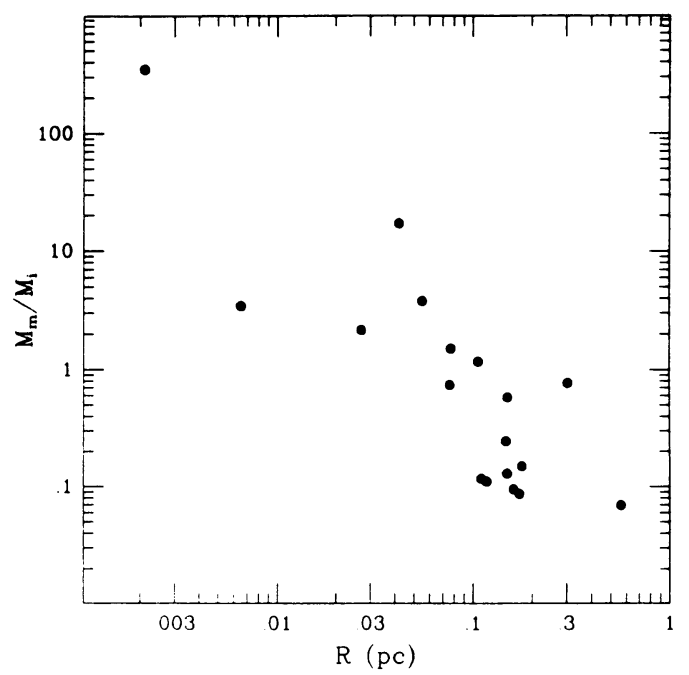

Fig. 1. The molecular/ionized mass ratio versus nebular radius (Huggins \& Healy 1989)

Other nearby $\mathrm{PNe}$ which are not seen in $\mathrm{CO}$ or $\mathrm{H}_{2}$ emission probably lose their molecular envelopes early in crossing the H-R diagram. Some of these are seen in atomic lines, but the systematics of their evolution remain to be sorted out.

\section{Concluding Remarks}

The main conclusions that can be made from the results described here can be summarized as follows. i) The presence of neutral envelopes around many PNe firmly links PN formation with mass loss by AGB stars and proto-PNe. ii) The neutral envelopes provide interesting laboratories in which to study highly unusual physical conditions and a unique chemistry. iii) The envelopes are found to play a key role in the evolution of a broad range of PNe, including many of the most familiar objects, for a large part of their observable lifetimes. In view of the fact that this field is still at a relatively early stage of development, further work is likely to prove very worthwhile.

Acknowledgements. It is a pleasure to acknowledge collaborative programs with Drs. R. Bachiller, P. Cox, T. Forveille, and A. Omont which form the basis for part of this paper. This work was supported in part by the National Science Foundation.

\section{References}

Bachiller, R., Planesas, P., Martin-Pintado, J., Bujarrabal, V., Tafalla, M., 1989a, A\&A, 210, 366

Bachiller, R., Bujarrabal, V., Martin-Pintado, J., Gomez-Gonzalez, J., 1989b, $\mathrm{A} \& \mathrm{~A}, 218,252$ 
Bachiller, R., Huggins, P.J., Cox, P., Forveille, T., 1991, A\&A, 247, 525

Bachiller, R., Huggins, P.J., Cox, P., Forveille, T., 1992, A\&A, in press

Bujarrabal, V., Gomez-Gonzalez, J., Bachiller, R., Martin-Pintado, J., 1988, A\&A, 204, 242

Bieging, J.H., Wilner, D., Thronson, H.A., 1991, ApJ, 379, 271

Black, J.H., 1983, in: Planetary Nebulae, ed. D.R. Flower, Reidel, Dordrecht, p. 91

Cox, P., Huggins, P.J., Bachiller, R., Forveille, T., 1991, A\&A, 250, 533

Cox, P., Omont, A., Huggins, P.J., Bachiller, R., Forveille, T., 1992, A\&A, in press

Deguchi, S., et al., 1992, ApJ, 392, 597

Dinerstein, H.L., 1991, PASP, 103, 861

Dinerstein, H.L., Haas, M.R., Werner, M.W., 1991, BAAS, 23, 915

Ellis, H.B., Werner, M.W., 1985, in: Mass Loss From Red Giants, eds. M. Morris \& B. Zuckerman, Reidel, Dordrecht, p. 309

Forveille, T., Huggins, P.J., 1991, A\&A, 248, 599

Greenhouse, M.A., Hayward, T.L., Thronson, H.A., 1988, ApJ, 325, 604

Gussie, G., 1992, private communicaton

Healy, A.P., Huggins, P.J., 1990, AJ, 100, 511

Howe, D.A., Millar, T.J., Williams, D.A., 1992, MNRAS, 255, 217

Huggins, P.J., 1992, in: Mass Loss on the AGB and Beyond, ed. H. Schwarz, ESO, in press

Huggins, P.J., Healy, A.P., 1989, ApJ, 346, 201

Huggins, P.J., Bachiller, R., Cox, P., Forveille, T., 1992a, in preparation

Huggins, P.J., Bachiller, R., Cox, P., Forveille, T., 1992b, ApJL, submitted

Jaminet, P.A., et al., 1991, ApJ, 380, 461

Likkel, L., 1992, ApJL, submitted

Neri, R., et al., 1992, these proceedings

Payne, H.E., Phillips, J.A., Terzian, Y., 1988, ApJ, 326, 368

Pottasch, S.R., 1980, A\&A, 89, 336

Rodriguez, L.F., 1989, in: Planetary Nebulae, ed. S. Torres-Peimbert, Kluwer, Dordrecht, p. 129

Sahai, R., Wootten, A., Clegg, R.E.S., 1990b, A\&A, 234, L1

Sahai, R., Wootten, A., Schwarz, H.E., Clegg, R.E.S., 1991, A\&A, 251, 560

Schwarz, H.E., 1992, A\&A, in press

Seaquist, E.R., Plume, R., Davis, L.E., 1991, ApJ, 367, 200

Sheperd, M.C., Cohen, R.J., Gaylard, M.J., West, M.E., 1990, Nature, 344, 522

Shibata, K.M., et al., 1992, these proceedings

Taylor, A.R., Gussie, G.T., Goss, W.M., 1989, ApJ, 340, 932

Taylor, A.R., Gussie, G.T., Pottasch, S.R., 1990, ApJ, 351, 515

Tielens, A.G.G.M., 1992, these proceedings

Walsh, J.R., Meaburn, J., 1987, MNRAS, 224, 885

Webster, B.L., Payne, P.W., Storey, J.W.V., Dopita, M.A., 1988, MNRAS, 235, 533

Zijlstra, A.A., et al., A\&A, 217, 157

Zuckerman, B., Gatley, I., 1988, ApJ, 324, 501

Zuckerman, B., Kastner, J.H., Balick, B., Gatley, I., 1990, ApJ, 356, L59 